\title{
Analysis of the Reynolds Equation for Lubrication in Case of Pressure-Dependent Viscosity
}

\author{
Eduard Marušić-Paloka and Sanja Marušić \\ Department of Mathematics, University of Zagreb, Bijenička Cesta 30, 10000 Zagreb, Croatia \\ Correspondence should be addressed to Eduard Marušić-Paloka; emarusic@math.hr
}

Received 30 November 2012; Revised 5 March 2013; Accepted 10 March 2013

Academic Editor: Jyh-Hong Chou

Copyright (C) 2013 E. Marušić-Paloka and S. Marušić. This is an open access article distributed under the Creative Commons Attribution License, which permits unrestricted use, distribution, and reproduction in any medium, provided the original work is properly cited.

\begin{abstract}
We study the Reynolds equation, describing the ow of a lubricant, in case of pressure-dependent viscosity. First we prove the existence and uniqueness of the solution. Then, we study the asymptotic behavior of the solution in case of periodic roughness via homogenization method. Some interesting nonlocal effects appear due to the nonlinearity.
\end{abstract}

\section{Introduction}

The Reynolds equations [1] describe the flow of a thin film of lubricant separating two rigid surfaces in relative motion. Controlling the flow of lubricant is an important engineering issue since inappropriate lubrication would increase the friction and wear, finally resulting in degrading the performance of the device. In his classical paper from 1848 [2] Stokes predicted that the viscosity of the fluid can depend on the pressure. Those effects for various liquids have been measured in many engineering papers, starting from the beginning of the 20th century (see, e.g., [3]). That effect is usually neglected as it becomes important only in case of high pressure. Most fluid-lubricated bearings operate with high pressure and in such a flow regime the dependence of the viscosity on the pressure becomes important. According to Szeri [4] the idea of pressure-dependent viscosity was introduced in lubrication theory by Gatcombe in 1945 [5]. Several models have been used to describe that relation since. The most popular is probably the exponential law

$$
\mu=\mu_{0} \exp (\alpha p)
$$

usually called the Barus formula [6]. Here $\mu_{0}$ and $\alpha$ are the constants depending on the lubricant. The formula seems to be reasonable for mineral oil, unless the pressure is very high (larger then $0.5 \mathrm{MPa}$ ). The coefficient $\alpha$ typically ranges between 1 and $10^{-8}$. The lower end of the range corresponds to paraffinic and the upper end corresponds to the naphthenic oils (see Jones [7]). That formula is still frequently used by engineers. The simplest viscosity-pressure relation is given by the the power law

$$
\mu(p)=\alpha|p|^{\gamma} .
$$

In case of the two above-mentioned laws explicit solutions of the equations of motion, for some particular situations like unidirectional and plane-parallel flows, were found in [8]. Discussion on other possibilities for the viscosity-pressure formula and some historical remarks on the subject can be found in the same paper. Several engineering papers can be found discussing other possible laws and their consistency. We mention, for instance, $[9,10]$.

We do not make any assumption on the particular form of the function $p \mapsto \mu(p)$. Some technical assumptions, like smoothness, will be needed for the proofs.

We study the stationary version of the Reynolds equation. Unless the velocity of relative motion is time dependent, steady approximation is reasonable in most cases (see, e.g., [4, Chapter 2.2]).

Our first goal is to prove that the problem is well posed. Secondly, we investigate the asymptotic behavior of the solution in case of periodically distributed asperities. Using the homogenization approach, we find the macroscopic Reynolds pressure. Interesting nonlocal effects appear due to the nonlinearity caused by the pressure-dependent viscosity. 


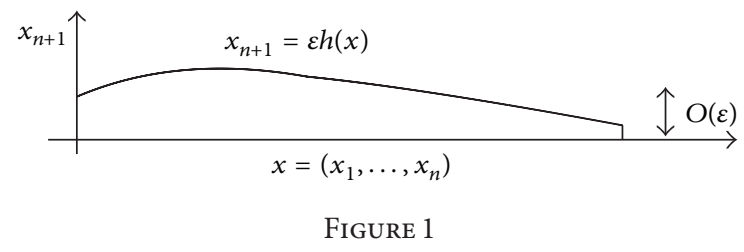

\section{Position of the Problem}

The fluid domain is bounded by two rigid surfaces. The simplified mathematical model can be written in the following form. Let $\mathcal{O} \subset \mathbf{R}^{n}$ be a bounded domain and let $h: \overline{\mathcal{O}} \rightarrow \mathbf{R}$ be a bounded, strictly positive smooth function such that

$$
0<h_{0} \leq h(x) \leq h_{1} .
$$

Function $h$ describes the shape of the slide. By $\varepsilon \ll 1$ we denote a very small parameter representing the domain thickness. Using the shape function $h$ we define the fluid domain (Figure 1) by

$$
\begin{aligned}
\Omega_{\varepsilon}=\{X & =\left(x, x_{n+1}\right) \in \mathbf{R}^{n+1} ; \\
x & \left.=\left(x_{1}, \ldots, x_{n}\right) \in \mathcal{O}, 0<x_{n+1}<\operatorname{ch}(x)\right\} .
\end{aligned}
$$

We then consider the stationary flow through a domain $\Omega_{\varepsilon}$. We want to describe the situation with a lower-dimensional model. The velocity of the relative motion of two surfaces is the constant vector denoted by $\mathbf{V}=v_{1} \mathbf{e}_{1}+\cdots+v_{n} \mathbf{e}_{n}$. The unknowns in the model are $u$ (the velocity) and $p$ (the pressure). We recall that the stationary motion of the incompressible viscous laminar flow is governed by the stationary Navier-Stokes equations. Thus we write the following system:

$$
\begin{gathered}
-\operatorname{div}[\mu(p) \mathbf{D u}]+(\mathbf{u} \nabla) \mathbf{u}+\nabla p=0, \quad \operatorname{div} \mathbf{u}=0 \quad \text { in } \Omega_{\varepsilon}, \\
\mathbf{u}=\mathbf{V} \quad \text { for } x_{n+1}=0, \quad \mathbf{u}=0 \quad \text { for } x_{n+1}=\varepsilon h(x),
\end{gathered}
$$

where $\mathbf{D u}=(1 / 2)\left(\nabla \mathbf{u}+\nabla \mathbf{u}^{t}\right)$ is the symmetric part of the velocity gradient. It is important to notice that in such system the pressure is not defined only up to a constant, as in the classical Navier-Stokes system with constant viscosity. Under certain technical assumptions, if the given data are not too large, the existence of the solution for such system was discussed in $[11,12]$. Neglecting the effects of inertia, we get the Stokes system with pressure-dependent viscosity

$$
\operatorname{div}[\mu(p) \mathbf{D u}]+\nabla p=0, \quad \operatorname{div} \mathbf{u}=0 \quad \text { in } \Omega_{\varepsilon},
$$

studied in [13].

If the thickness of the domain is small, the solution can be fairly approximated by the solution of the Reynolds equations $[4,14]$

$$
\begin{gathered}
\mathbf{v}\left(x, x_{n+1}\right) \\
=\frac{1}{12 \mu(p)} x_{n+1}\left(\varepsilon h-x_{n+1}\right) \nabla p(x)+\left(1-\frac{x_{n+1}}{\varepsilon h}\right) \mathbf{V} \\
\operatorname{div}\left(\int_{0}^{\varepsilon h} \mathbf{v} d x_{n+1}\right)=0
\end{gathered}
$$

Indeed, if we derive a formal asymptotic expansion of the solution to the system (5) in powers of $\varepsilon$, then the solution of the Reynolds equation (7) makes the first term of the expansion (see, e.g., [4]). Here and in the sequel the differential operators div and $\nabla$ are taken only with respect to $x$ variable; that is,

$$
\begin{gathered}
\operatorname{div} \mathbf{b}=\frac{\partial b_{1}}{\partial x_{1}}+\cdots+\frac{\partial b_{n}}{\partial x_{n}} \\
\nabla \phi=\frac{\partial \phi}{\partial x_{1}} \mathbf{e}_{1}+\cdots+\frac{\partial \phi}{\partial x_{n}} \mathbf{e}_{n} .
\end{gathered}
$$

It leads to an elliptic equation of the form

$$
\begin{gathered}
\operatorname{div}\left(\frac{h^{3}}{\mu(p)} \nabla p\right)=6 \mathbf{V} \cdot \nabla h \text { in } \mathcal{O} \\
p=q \text { on } \partial \mathcal{O} .
\end{gathered}
$$

The goal of this paper is to study that equation.

We assume that the function $p \mapsto \mu(p)$ is of class $C^{1}(\mathbf{R})$ and $\mu>0$ for any value of $p$. In real life the viscosity increases with pressure, but such an assumption is not necessary for our study.

\section{Existence of the Solution}

3.1. Transformed Equation. Equation (10) is a quasilinear elliptic PDE, but it can be linearized by simple trick. To do so we rewrite the equation using the function

$$
M_{\sigma}(p)=\int_{\sigma}^{p} \frac{d t}{\mu(t)} .
$$

We choose $\sigma \leq q$. Function $M_{\sigma}$ is strictly increasing, since $M_{\sigma}^{\prime}(p)=(1 / \mu(p))>0$ and thus it is bijective. Furthermore $M_{\sigma}(p)$ has the same sign as $p-\sigma$; that is, for $p>\sigma$ we have $M_{\sigma}(p)>0$, for $p<\sigma$ obviously $M_{\sigma}(p)<0$, and finally $M_{\sigma}(p)=0$, if and only if $p=\sigma$.

We introduce the new unknown function

$$
w(x)=M_{\sigma}(p(x))=\int_{\sigma}^{p(x)} \frac{d t}{\mu(t)} .
$$

At this point we assume that the integral $\int_{0}^{-\infty}(d t / \mu(t))$ is divergent, that is,

$$
\int_{-\infty}^{0} \frac{d t}{\mu(t)}=+\infty
$$

As a consequence

$$
\lim _{\sigma \rightarrow-\infty} M_{\sigma}(p)=+\infty, \quad \forall p \in \mathbf{R},
$$

as well as

$$
\lim _{p \rightarrow-\infty} M_{\sigma}(p)=-\infty, \quad \forall \sigma \in \mathbf{R} .
$$


Deriving (13) we obtain

$$
\frac{1}{\mu(p)} \nabla p=\nabla w
$$

and the problem can be written as

$$
\begin{gathered}
\operatorname{div}\left(h^{3} \nabla w\right)=6 \mathbf{V} \cdot \nabla h \quad \text { in } \mathcal{O}, \\
w=M_{\sigma}(q) \text { on } \partial \mathcal{O} .
\end{gathered}
$$

That is a linear elliptic equation for $w$ and it has a unique solution. To get the existence and uniqueness of the solution we quote Theorem 8.34 from classical book of Gilbarg and Trudinger [15]. For simplicity, here and in the sequel, we assume that $q$ and, consequently, $M_{\sigma}(q)$ are defined on whole $\overline{\mathcal{O}}$. We combine that with the maximum principle from the appendix, and it gives the following.

Theorem 1. Under the assumption that the boundary $\partial \mathcal{O}$ is of class $C^{1, \alpha}$ and that $h \in C^{\alpha}(\overline{\mathcal{O}}), q \in C^{1, \alpha}(\overline{\mathcal{O}})$ the problem (18), (19) has a unique solution

$$
w \in C^{1, \alpha}(\overline{\mathcal{O}})
$$

Furthermore

$$
w(x) \leq M(\bar{q})+\mathscr{Z},
$$

where

$$
\bar{q}=|q|_{L^{\infty}(\partial \omega)}
$$

and $\mathscr{Z}=0$ if $\mathbf{V} \cdot \nabla h<0$. Otherwise

$$
\mathscr{Z}=\left\{\begin{array}{lc}
6|\mathbf{V}|\left[\int_{0}^{1} \frac{d t}{h(t)^{2}}-\frac{h_{0}^{3}}{h_{1}^{5}}\right]+\left(\frac{h_{0}}{h_{1}}\right)^{3} \int_{q_{0}}^{q_{1}} \frac{d s}{\mu(s)} \\
3^{(8 / 5)}\left(\frac{3}{2}\right)^{(28 / 5)} \frac{(2 \pi)^{(1 / 4)}}{h_{0}^{3}} & \text { if } n=1 \\
\times d\left(6|\mathbf{V}| h_{1}|\mathcal{O}|^{1 / 5}+h_{1}^{3}\left|\nabla M_{\sigma}(q)\right|_{L^{5}(\mathcal{O})}\right)|\mathcal{O}|^{(7 / 5)} & \text { if } n=2,
\end{array}\right.
$$

with $d=\operatorname{diam} \mathcal{O}$.

Proof. The existence follows directly from Theorem 8.34. from Gilbarg and Trudinger [15]. If $\mathbf{V} \cdot \nabla h<0$, then (21) follows directly from the weak maximum principle (see, e.g., [15]). In case $n=1$ the problem can be solved by quadratures and the solution given by (25) can be easily estimated to get (23). In the remaining case $n=2$ (21) follows from the special variant of the maximum principle proved in the appendix.

Remark 2. In case $n=1(18)$ is an ODE (we take $\mathcal{O}=] 0,1[$ and $q_{0}>q_{1}$, without losing generality)

$$
\begin{aligned}
& \left.\left(h^{3} w^{\prime}\right)^{\prime}=6 V h^{\prime} \quad \text { in }\right] 0,1[, \\
& w(i)=M_{\sigma}\left(q_{i}\right) \quad \text { for } i=0,1,
\end{aligned}
$$

and it can be solved by quadratures

$$
\begin{array}{r}
w(x)=M_{\sigma}\left(q_{0}\right) \\
+\int_{0}^{x} \frac{1}{h(t)^{3}}\left[6 V\left(h(t)-\frac{\int_{0}^{1}\left(d r / h(r)^{2}\right)}{\int_{0}^{1}\left(d s / h(s)^{3}\right)}\right)\right. \\
\left.+\frac{M_{\sigma}\left(q_{1}\right)-M_{\sigma}\left(q_{0}\right)}{\int_{0}^{1}\left(d s / h(s)^{3}\right)}\right] d t .
\end{array}
$$

3.2. Back to the Original Equation. Now, our goal is not to find the auxiliary function $w$ but to find the pressure $p$. Since we have introduced $w$ as

$$
w=M_{\sigma}(p)
$$

we should have $p=M_{\sigma}^{-1}(w)$. In order to do so we have to make sure that $w(x) \in \operatorname{Im} M_{\sigma}$ for any $x \in \overline{\mathcal{O}}$. Since $M_{\sigma}$ is strictly increasing and we have assumed that (14) holds, if we define

$$
M_{\sigma}^{+}=\lim _{s \rightarrow+\infty} M_{\sigma}(s)=\int_{\sigma}^{+\infty} \frac{d s}{\mu(s)},
$$

due to (16) we obviously have for any $p \in \mathbf{R}$

$$
-\infty=\lim _{s \rightarrow-\infty} M_{\sigma}(s) \leq M_{\sigma}(p) \leq M_{\sigma}^{+} .
$$

Thus

$$
\left.\operatorname{Im} M_{\sigma}=\right]-\infty, M_{\sigma}^{+}[
$$

So, to fulfill the condition $w(x) \in \operatorname{Im} M_{\sigma}$ we need to have

$$
w(x) \leq M_{\sigma}^{+}, \quad \forall x \in \overline{\mathcal{O}} .
$$

That condition is not necessarily fulfilled. In view of (21) that condition reduces to

$$
\mathscr{Z} \leq \int_{\bar{q}}^{+\infty} \frac{d t}{\mu(t)},
$$

where $\mathscr{Z}=\mathscr{Z}(\mathbf{V}, h, \mathcal{O}, \mu, q)$ is defined by (23).

We have proved the following theorem.

Theorem 3. Suppose that the conditions of Theorem 1 hold, and that in addition (31) is fulfilled. Then $p=M_{\sigma}^{-1}(w)$ is the unique solution of (10) and (11).

Remark 4. It is important to notice that even though $w$ does depend on $\sigma$, the effective pressure $p$ does not. For the purpose of this remark, we denote $H_{\sigma}(w)=M_{\sigma}^{-1}(w)$ to stress the dependence on the parameter $\sigma$ which is of interest here. We start by

$$
p=H_{\sigma}(w) .
$$


It is obvious from the definition of $M_{\sigma}$ that

$$
\frac{\partial M_{\sigma}}{\partial p}(p)=\frac{1}{\mu(p)}, \quad \frac{\partial M_{\sigma}}{\partial \sigma}(p)=-\frac{1}{\mu(\sigma)} .
$$

As $M_{\sigma}\left(H_{\sigma}(w)\right)=w$, deriving with respect to $\sigma$ we arrive at

$$
\begin{aligned}
\frac{\partial H_{\sigma}}{\partial \sigma}(w) & =-\frac{\left(\partial M_{\sigma} / \partial \sigma\right)(p)}{\left(\partial M_{\sigma} / \partial p\right)(p)} \\
& =\frac{\mu(p)}{\mu(\sigma)}=\frac{\mu\left(H_{\sigma}(w)\right)}{\mu(\sigma)} .
\end{aligned}
$$

Deriving (13) we get $(\partial w / \partial \sigma)=-1 / \mu(\sigma)$. Using the rule for deriving the inverse function, we have

$$
\frac{\partial H_{\sigma}}{\partial w}(w)=\mu(p)=\mu\left(H_{\sigma}(w)\right) .
$$

Thus

$$
\begin{aligned}
\frac{\partial p}{\partial \sigma} & =\frac{\partial H_{\sigma}}{\partial w}(w) \frac{\partial w}{\partial \sigma}(\sigma)+\frac{\partial H_{\sigma}}{\partial \sigma}(w) \\
& =-\frac{\mu\left(H_{\sigma}(w)\right)}{\mu(\sigma)}+\frac{\mu\left(H_{\sigma}(w)\right)}{\mu(\sigma)}=0 .
\end{aligned}
$$

\section{Homogenization}

In this section we want to study the effects of rugosities of surfaces on lubrication process. The idea of finding the macroscopic effects of roughness on lubrication process, via homogenization, is quite old and well studied. Case of constant viscosity for incompressible and compressible flows as well as non-Newtonian, deformation dependent, viscosities were investigated. The subject was treated by several authors and we here mention [16-18]. The case of pressure-dependent viscosity brings some new interesting nonlocal effects.

We assume that the function $h$, describing the form of the fluid domain, is periodic with small period $1 / m$, with $m \in \mathbf{N}$. To stress that dependence we denote it by $h_{m}$. More precisely, we denote by $Y=] 0,1{ }^{n}, n=1,2$, the period. We further assume that $h: \mathbf{R}^{n} \rightarrow\left[d_{0},+\infty\left[, d_{0}>0\right.\right.$ is periodic with period $Y$ and smooth. Then we take $h_{m}$ of the form

$$
h_{m}(x)=h(m x) \text {. }
$$

Thus, the function $h$ describes the form of periodically distributed rugosities.

To emphasize that the relative velocity of bearing surfaces $\mathbf{V}$ is large, we assume that it also depends on $m$, the same parameter that is taken for description of rugosities. In that case our equation reads

$$
\operatorname{div}\left(\frac{h_{m}^{3}}{\mu\left(p_{m}\right)} \nabla p_{m}\right)=6 \mathbf{V}_{m} \cdot \nabla h_{m} \text { in } \mathcal{O} .
$$

4.1. One-Dimensional Case. If $n=1$, the above problem is posed on an interval $\mathcal{O}=] 0,1[$. With an appropriate boundary condition

$$
p_{m}(0)=q_{0}, \quad p_{m}(1)=q_{1} \text {. }
$$

It forms a boundary value problem for nonlinear ODE:

$$
\left(\frac{h_{m}^{3}}{\mu\left(p_{m}\right)} p_{m}^{\prime}\right)^{\prime}=6 V_{m} h_{m}^{\prime} \text { in } \mathcal{O}
$$

To study the asymptotic behavior of the solution with respect to $m$ we linearize the problem using the transformation $w_{m}=$ $M_{\sigma}\left(p_{m}\right)$. To simplify, in this section we choose $\sigma=q_{0}$ and, dropping the index $\sigma$ in $M_{\sigma}$ and $M_{\sigma}^{+}$, we denote

$$
M(p)=\int_{q_{0}}^{p} \frac{d t}{\mu(t)}, \quad M^{+}=\int_{q_{0}}^{+\infty} \frac{d t}{\mu(t)} .
$$

Theorem 5. Let

$$
\begin{aligned}
& \chi_{0}(y)= 6\left(\int_{0}^{1} \frac{d s}{h(s)^{3}}\right)^{-1} \\
& \times\left[\left\langle\frac{1}{h^{3}}\right\rangle \int_{0}^{y} \frac{d v}{h(v)^{2}}-\left\langle\frac{1}{h^{2}}\right\rangle \int_{0}^{y} \frac{d v}{h(v)^{3}}\right], \\
&\langle\cdot\rangle=\int_{0}^{1} \cdot d y,
\end{aligned}
$$

and let $H=M^{-1}$. Suppose that there exists a limit

$$
V=\lim _{m \rightarrow \infty} \frac{V_{m}}{m}
$$

and that, for $m$ large enough and $M^{+}$defined in (27), the following condition holds:

$$
\frac{b_{3}^{m}(x)}{b_{3}^{m}(1)} M\left(q_{1}\right)+6 V_{m} \frac{b_{3}^{m}(1) b_{2}^{m}(x)-b_{3}^{m}(x) b_{2}^{m}(1)}{b_{3}^{m}(1)} \leq M^{+},
$$

with

$$
b_{\alpha}^{m}(x)=\int_{0}^{x} \frac{d t}{h_{m}(t)^{\alpha}}, \quad \alpha=2,3 .
$$

Then

$$
\begin{array}{r}
p_{m} \rightarrow p_{0}=\int_{0}^{1} H\left(x M\left(q_{1}\right)+V \chi_{0}(y)\right) d y \\
\text { weak }^{*} \text { in } L^{\infty}(0,1) .
\end{array}
$$

Proof. Equation (40), with boundary conditions (39), can be solved by reduction to quadratures, after the substitution

$$
w_{m}=M\left(p_{m}\right)
$$

with $M_{\sigma}$ strictly increasing function defined by (12). The problem for $w_{m}$ now reads

$$
\begin{gathered}
\left.\left(h_{m}^{3} w_{m}^{\prime}\right)^{\prime}=6 V_{m} h_{m}^{\prime} \quad \text { in }\right] 0,1[ \\
w_{m}(0)=M\left(q_{0}\right)=0, \quad w_{m}(1)=M\left(q_{1}\right) .
\end{gathered}
$$


It is easy to see that (49) has a unique solution given by (25). Since $M\left(q_{0}\right)=0,(25)$ now reduces to

$$
w_{m}=\frac{b_{3}^{m}(x)}{b_{3}^{m}(1)} M_{\sigma}\left(q_{1}\right)+6 V_{m} \frac{b_{3}^{m}(1) b_{2}^{m}(x)-b_{3}^{m}(x) b_{2}^{m}(1)}{b_{3}^{m}(1)},
$$

where, for $\alpha=2,3$,

$$
\begin{aligned}
b_{\alpha}^{m}(x) & =\int_{0}^{x} \frac{d t}{h_{m}(t)^{\alpha}} \\
& =\frac{1}{m} \int_{0}^{m x} \frac{d s}{h(s)^{\alpha}} \longrightarrow x \int_{0}^{1} \frac{d s}{h(s)^{\alpha}}, \\
& \text { as } m \longrightarrow+\infty .
\end{aligned}
$$

Now $p_{m}$, the solution to the problem (40), exists if (45) is fulfilled. The second term in (50) thus obviously tends to $x M\left(q_{1}\right)$, as $m \rightarrow+\infty$. The last term is more interesting. The denominator tends to

$$
\left\langle\frac{1}{h^{3}}\right\rangle=\int_{0}^{1} \frac{d s}{h(s)^{3}} .
$$

As for its numerator, we have

$$
\begin{aligned}
V_{m}[ & \left.b_{3}^{m}(1) b_{2}^{m}(x)-b_{3}^{m}(x) b_{2}^{m}(1)\right] \\
& =V_{m}\left[\int_{0}^{1} \int_{0}^{x}\left(\frac{1}{h_{m}(t)^{3} h_{m}(s)^{2}}-\frac{1}{h_{m}(t)^{2} h(s)_{m}^{3}}\right) d s d t\right] \\
& =\frac{V_{m}}{m}\left(\left\langle\frac{1}{h^{3}}\right\rangle \int_{0}^{m x} \frac{d v}{h(v)^{2}}-\left\langle\frac{1}{h^{2}}\right\rangle \int_{0}^{m x} \frac{d v}{h(v)^{3}}\right) .
\end{aligned}
$$

Suppose that

$$
V=\lim _{m \rightarrow+\infty} \frac{V_{m}}{m}
$$

and denote

$$
G(y)=\left\langle\frac{1}{h^{3}}\right\rangle \int_{0}^{y} \frac{d v}{h(v)^{2}}-\left\langle\frac{1}{h^{2}}\right\rangle \int_{0}^{y} \frac{d v}{h(v)^{3}} .
$$

Obviously the function $G$ is periodic with period 1 so that, due to the standard periodicity lemma (see, e.g., [19]), as $m \rightarrow$ $+\infty$,

$$
G(m x) \rightarrow\langle G\rangle=\int_{0}^{1} G(y) d y, \quad \text { weak }^{*} \text { in } L^{\infty}(0,1) .
$$

By direct computation

$$
\begin{aligned}
\langle G\rangle & =\left\langle\frac{y}{h^{3}}\right\rangle\left\langle\frac{1}{h^{2}}\right\rangle-\left\langle\frac{1}{h^{3}}\right\rangle\left\langle\frac{y}{h^{2}}\right\rangle \\
& =\iint_{0}^{1} \frac{s-t}{h(s)^{3} h(t)^{2}} d s d t .
\end{aligned}
$$

Thus

$$
V_{m}\left[b_{3}^{m}(1) b_{2}^{m}(x)-b_{3}^{m}(x) b_{2}^{m}(1)\right] \rightarrow V\langle G\rangle .
$$

Now, denoting

$$
\chi_{0}(y)=\frac{G(y)}{b_{3}(1)}
$$

we have

$$
w_{m} \approx x M\left(q_{1}\right)+V \chi_{0}(m x),
$$

and thus

$$
w_{m} \rightarrow w_{0}=x M\left(q_{1}\right)+V\left\langle\chi_{0}\right\rangle \text { weak }{ }^{*} \text { in } L^{\infty}(0,1) .
$$

However, we are not interested in convergence of the auxiliary function $w_{m}$ but in the convergence of the pressure $p_{m}$. Since (45) is assumed to be true, we can define $p_{m}=$ $H\left(w_{m}\right)$, where $H=M^{-1}$, and we have

$$
\begin{array}{r}
p_{m} \rightarrow p_{0}=\int_{0}^{1} H\left(x M\left(q_{1}\right)+V \chi_{0}(y)\right) d y \\
\text { weak }^{*} \text { in } L^{\infty}(0,1) .
\end{array}
$$

Deriving the expression on the right-hand side, we obtain the effective pressure drop in the form

$$
\begin{aligned}
p_{0}^{\prime}(x) & =M\left(q_{1}\right) \int_{0}^{1} \mu\left(x M\left(q_{1}\right)+V \chi_{0}(y)\right) d y \\
& =\int_{q_{0}}^{q_{1}} \frac{d \tau}{\mu(\tau)} \int_{0}^{1} \mu\left(x M\left(q_{1}\right)+V \chi_{0}(y)\right) d y \\
& =\int_{q_{0}}^{q_{1}} \frac{d \tau}{\mu(\tau)} \int_{0}^{1} \mu\left(x \int_{q_{0}}^{q_{1}} \frac{d s}{\mu(s)}+V \chi_{0}(y)\right) d y
\end{aligned}
$$

As we can see, the pressure drop is not constant, as for the Newtonian flow. The interesting effect appears if $V \neq 0$ because, in that case, the expressions for the pressure and for the pressure drop are nonlocal due to the integral with respect to $y$. That phenomenon is entirely due to the fact that the viscosity is depending on the pressure.

4.2. Two-Dimensional Case. We suppose here that the function $h_{m}$ is constructed from positive, smooth, $Y$-periodic function $h: \mathbf{R}^{2} \rightarrow\left[d_{0},+\infty\left[, d_{0}>0, Y=\right] 0,1\left[^{2}\right.\right.$ in the same way as before, that is, by taking

$$
h_{m}(x)=h(m x) \text {. }
$$

We have seen in the previous section that interesting effects happen only if we assume that

$$
\mathbf{V}_{m}=m \mathbf{V}
$$

In that case our equation reads

$$
\operatorname{div}\left(\frac{h_{m}^{3}}{\mu\left(p_{m}\right)} \nabla p_{m}\right)=6 \mathbf{V}_{m} \cdot \nabla h_{m} \text { in } \mathcal{O} \text {. }
$$


As we did in the existence analysis and in the previous section, we linearize the equation by substitution

$$
w_{m}=M_{\sigma}\left(p_{m}\right)
$$

where the function $M_{\sigma}$ is defined by (12). Now $w_{m}$ satisfies

$$
\operatorname{div}\left(h^{3} M \nabla w_{m}\right)=6 \mathbf{V}_{m} \cdot \nabla h_{m} \text { in } \mathcal{O} .
$$

We postulate the asymptotic expansion in the form

$$
\begin{aligned}
w_{m}(x) \approx & w_{0}(x, y)+\frac{1}{m} w_{1}(x, y) \\
& +\frac{1}{m^{2}} w_{2}(x, y)+\cdots, \quad y=m x .
\end{aligned}
$$

All functions are assumed to be $Y$-periodic in $y$ variable.

Plugging that in (69) and collecting, formally, terms with equal powers of $m$, we get

$$
\begin{aligned}
& m^{2}: \operatorname{div}_{y}\left(h^{3} \nabla_{y} w_{0}\right)=6 \mathbf{V} \cdot \nabla_{y} h \\
& m: \operatorname{div}_{y}\left(h^{3} \nabla_{y} w_{1}\right)+\operatorname{div}_{y}\left(h^{3} \nabla_{x} w_{0}\right)+h^{3} \operatorname{div}_{x} \nabla_{y} w_{0}=0 \\
& 1: \operatorname{div}_{y}\left(h^{3} \nabla_{y} w_{2}\right)+\operatorname{div}_{y}\left(h^{3} \nabla_{x} w_{1}\right)+h^{3} \operatorname{div}_{x} \nabla_{y} w_{1} \\
& \quad+h^{3} \Delta_{x} w_{0}=0 .
\end{aligned}
$$

Denoting

$$
V=|\mathbf{V}|, \quad \mathbf{V}_{0}=\frac{\mathbf{V}}{V}
$$

we have

$$
\begin{gathered}
w_{0}(x, y)=v_{0}(x)+V \chi_{0}(y), \\
\operatorname{div}_{y}\left(h^{3} \nabla_{y} \chi_{0}\right)=6 \mathbf{V}_{0} \cdot \nabla_{y} h \quad \text { in } Y, \\
w_{1}(x, y)=\sum_{k=1}^{2} \chi_{k}(y) \frac{\partial v_{0}}{\partial x_{k}}(x)+v_{1}(x), \\
\operatorname{div}_{y}\left(h^{3} \nabla_{y}\left(\chi_{k}+y_{k}\right)\right)=0, \\
\sum_{k, \ell=1}^{2} \mathbf{a}_{k \ell} \frac{\partial^{2} v_{0}}{\partial x_{k} \partial x_{\ell}}=0 \quad \text { in } \mathcal{O}, \\
\mathbf{a}_{k \ell}=\int_{Y} h^{3} \frac{\partial}{\partial y_{\ell}}\left(\chi_{k}+y_{k}\right) d y .
\end{gathered}
$$

Remark 6. The same computation can be done in one-dimensional case and it gives

$$
\begin{gathered}
\left(h^{3} \chi_{0}^{\prime}\right)^{\prime}=6 h^{\prime} \Longrightarrow \chi_{0}=6 \int_{0}^{y} \frac{d t}{h(t)^{2}}+C_{0} \int_{0}^{y} \frac{d t}{h(t)^{3}}+C_{1} \\
v_{0}=x M\left(q_{1}\right) .
\end{gathered}
$$

Constants $C_{0}, C_{1}$ are chosen in a way that boundary conditions $\chi_{0}(0)=\chi_{0}(1)=0$ are met, and it follows that

$$
C_{1}=0, \quad C_{0}=-6\left(\int_{0}^{1} \frac{d t}{h(t)^{3}}\right)^{-1} \int_{0}^{1} \frac{d t}{h(t)^{2}} .
$$

Then

$$
\begin{aligned}
w_{m} \approx & w_{0}(x, m x) \\
= & v_{0}(x)+\chi_{0}(m x) \\
= & x M_{\sigma}\left(q_{1}\right) \\
& +6 V\left[\int_{0}^{m x} \frac{d t}{h(t)^{2}}-\left(\int_{0}^{1} \frac{d t}{h(t)^{3}}\right)^{-1}\right. \\
& \left.\times \int_{0}^{1} \frac{d t}{h(t)^{2}} \int_{0}^{m x} \frac{d t}{h(t)^{3}}\right] \\
= & x M_{\sigma}\left(q_{1}\right) \\
& +6 V \frac{b_{2}^{m}(x) b_{3}(1)-b_{2}(1) b_{3}^{m}(x)}{b_{3}(1)} \\
= & x M_{\sigma}\left(q_{1}\right)+V \chi_{0}(m x) .
\end{aligned}
$$

That is a very good approximation of our exact solution (50). It is important to notice that the choice of constants $C_{0}, C_{1}$ was determined from the exterior boundary condition. So, we should expect the same in two-dimensional case. However the treatment of boundary conditions in two-dimensional case is much more complicated and the boundary layer is to be expected.

The derived asymptotic expansion should be justified by proving the convergence. And we need the strong convergence (with corrector, of course) for $w_{m}$ in order to get the convergence for $p_{m}$. The form of the approximation

$$
w_{m} \approx v_{0}(x)+V \chi_{0}(m x)+\cdots .
$$

suggests that the boundary layer phenomenon should appear on the exterior boundary $\partial \mathcal{O}$ since $\chi_{0}$ term cannot satisfy the Dirichlet condition on $\partial \mathcal{O}$. To get the error estimate and the strong convergence we need to handle that boundary layer. Thus, at this point we simplify the domain and the boundary condition, in order to be able to avoid it. We assume that

$$
\begin{gathered}
\mathcal{O}=] 0,1[\times \mathbf{R}, \\
p_{\varepsilon}\left(0, x_{2}\right)=0, \quad p_{\varepsilon}\left(1, x_{2}\right)=q\left(x_{2}\right), \\
x_{2} \longmapsto p_{\varepsilon}\left(x_{1}, x_{2}\right) \text { is 1-periodic, } \\
h\left(y_{1}, y_{2}\right)=h\left(y_{1}\right), \\
q \text { is 1-periodic. }
\end{gathered}
$$

Now

$$
\begin{gathered}
w_{\varepsilon}=M_{\sigma}\left(p_{\varepsilon}\right), \quad \text { with } M_{\sigma}(p)=\int_{\sigma}^{p} \frac{d s}{\mu(s)} \Longrightarrow w_{\varepsilon}\left(0, x_{2}\right)=0 \\
w_{\varepsilon}\left(1, x_{2}\right)=M_{\sigma}\left(q\left(x_{2}\right)\right)
\end{gathered}
$$

and $x_{2} \mapsto w_{\varepsilon}\left(x_{1}, x_{2}\right)$ is 1-periodic. 
In that case we can compute $\chi_{0}$ and $\chi_{k}, k=1,2$, explicitly and we can impose exterior condition on $\chi_{0}$. Indeed $\chi_{0}$ is exactly the same as in the monodimensional case; that is, it is given by (73) and (75). Obviously $\chi_{2}=0$ so that

$$
\mathbf{a}_{22}=\int_{0}^{1} h(s)^{3} d s=\left\langle h^{3}\right\rangle, \quad \mathbf{a}_{12}=\mathbf{a}_{21}=0 .
$$

As for the last term

$$
\begin{gathered}
\chi_{1}=-y_{1}+\left(\int_{0}^{1} \frac{d s}{h(s)^{3}}\right)^{-1} \int_{0}^{y_{1}} \frac{d s}{h(s)^{3}}, \\
\mathbf{a}_{11}=\left(\int_{0}^{1} \frac{d s}{h(s)^{3}}\right)^{-1}=\frac{1}{\left\langle 1 / h^{3}\right\rangle} .
\end{gathered}
$$

Finally, the function $v_{0}$ satisfies the boundary value problem

$$
\begin{gathered}
\frac{1}{\left\langle 1 / h^{3}\right\rangle} \frac{\partial^{2} v_{0}}{\partial x_{1}^{2}}+\left\langle h^{3}\right\rangle \frac{\partial^{2} v_{0}}{\partial x_{2}^{2}}=0 \quad \text { in } \mathcal{O}, \\
v_{0}\left(0, x_{2}\right)=0, \\
v_{0}\left(1, x_{2}\right)=M_{\sigma}\left(q\left(x_{2}\right)\right), \quad v_{0} \text { is 1-periodic in } x_{2} .
\end{gathered}
$$

It can be solved using the Fourier method, and we get

$$
\begin{aligned}
v_{0}\left(x_{1}, x_{2}\right)= & \sum_{k=1}^{\infty} \operatorname{sh}\left(\sqrt{\left\langle h^{3}\right\rangle\left\langle h^{-3}\right\rangle} k \pi x_{1}\right) \\
& \times\left(\alpha_{k} \sin k \pi x_{2}+\beta_{k} \cos k \pi x_{2}\right), \\
\alpha_{k}= & 2 \int_{0}^{1} M_{\sigma}(q(t)) \sin k \pi t d t, \\
\beta_{k}= & 2 \int_{0}^{1} M_{\sigma}(q(t)) \cos k \pi t d t .
\end{aligned}
$$

Since the approximation

$$
\mathrm{w}_{m} \approx v_{0}(x)+V \chi_{0}\left(m x_{1}\right)+\frac{1}{m} \chi_{1}\left(m x_{1}\right) \frac{\partial v_{0}}{\partial x_{1}}(x)
$$

now satisfies the boundary conditions on $\partial \mathcal{O}$, it is easy to see that

$$
\left|w_{m}-\left(v_{0}(x)+V \chi_{0}(m x)\right)\right|_{L^{\infty}(\mathcal{O})} \leq C \frac{1}{m}
$$

follows from the maximum principle. Assuming that, for $m$ large enough,

$$
v_{0}(x)+V \chi_{0}(m x) \leq M^{+}
$$

we have

$$
\left|p_{m}-H\left(v_{0}(x)+V \chi_{0}(m x)\right)\right|_{L^{\infty}(\mathcal{O})} \longrightarrow 0 .
$$

Finally

$$
p_{m} \rightarrow p_{0}=\int_{0}^{1} H\left(v_{0}(x)+V \chi_{0}\left(y_{1}\right)\right) d y_{1} .
$$

We have proved that.
Theorem 7. Let $p_{m}$ be the solution to the problem (67), (79), and (80) and let $v_{0}, \chi_{0}$ be defined by (87) and (42), respectively. If (90) holds, then

$$
\begin{aligned}
& \left|p_{m}-H\left(v_{0}(x)+V \chi_{0}(m x)\right)\right|_{L^{\infty}(\mathcal{O})} \longrightarrow 0, \\
& p_{m} \rightarrow p_{0}=\int_{0}^{1} H\left(v_{0}(x)+V \chi_{0}\left(y_{1}\right)\right) d y_{1} .
\end{aligned}
$$

Remark 8. It is important to notice that $\pi_{0}=H\left(p_{0}\right)$ satisfies

$$
\sum_{i, k=1}^{2} \frac{\partial}{\partial x_{i}}\left(\frac{\mathbf{a}_{i k}}{\mu\left(\pi_{0}\right)} \frac{\partial \pi_{0}}{\partial x_{k}}\right)=0 \quad \text { in } \mathcal{O}, \pi_{0}=q \text { on } \partial \mathcal{O},
$$

and thus we would expect it to be the limit of $p_{m}$ in analogy with the linear case. However $p_{0} \neq \pi_{0}$.

If $V=\lim _{m \rightarrow \infty} m\left|\mathbf{V}_{m}\right|$ is small, we can expand $H\left(v_{0}(x)+\right.$ $\left.V \chi_{0}(m x)\right)$ in powers of $V$ and we get

$$
\begin{aligned}
p_{m}(x) & \approx H\left(v_{0}(x)+V \chi_{0}(m x)\right) \\
& =H\left(v_{0}(x)\right)+V H^{\prime}\left(v_{0}(x)\right) \chi_{0}(y)+O\left(V^{2}\right) \\
& =\pi_{0}(x)+V \mu\left(\pi_{0}(x)\right) \chi_{0}(y)+O\left(V^{2}\right) .
\end{aligned}
$$

Thus

$$
p_{0}=\pi_{0}+\mu\left(\pi_{0}\right)\left\langle\chi_{0}\right\rangle V+O\left(V^{2}\right) .
$$

It can be, formally, written as

$$
p_{m}(x) \approx \pi_{0}(x)+\frac{\left|\mathbf{V}_{m}\right|}{m} \mu\left(\pi_{0}(x)\right) \chi_{0}(m x)+O\left(\frac{\left|\mathbf{V}_{m}\right|^{2}}{m^{2}}\right) .
$$

\section{Appendix}

\section{The Maximum Principle}

Our goal is to derive maximum principles for the linear Reynolds equation, with sharp explicit constants, in order to solve the nonlinear Reynolds equation with pressuredependent viscosity. We assume, without losing generality, that $\mathbf{V}=(1 / 6) v \mathbf{i}$. Indeed, we can always choose the coordinate system in a way that the first coordinate axis $x$ has a direction of the velocity of relative motion $\mathbf{V}$.

The lower bound for $w(x, y)$ is of no interest, just the upper bound. Function $w(x, y)$ is the solution to the boundary value problem

$$
\begin{gathered}
\operatorname{div}\left(h^{3} \nabla w\right)=v \frac{\partial h}{\partial x} \quad \text { in } \mathcal{O} \subset \mathbf{R}^{2}, \\
w=M_{\sigma}(q) \quad \text { on } \partial \mathcal{O} .
\end{gathered}
$$

We assume that if $v(\partial h / \partial x)>0$, then $w$ cannot have a maximum point in the domain $\mathcal{O}$ and, thus,

$$
w(x, y) \leq \max _{(x, y) \in \partial \Theta} M_{\sigma}(q(x, y)) .
$$


However it is not realistic to assume that $(\partial h / \partial x)$ does not change the sign. To find the upper bound in the general case we use the procedure from the DeGiorgi theorem. The main result of the section is as follows.

Theorem A.1. Let $w$ be the solution to the problem (A.1). Then

$$
\begin{aligned}
& w(x, y) \leq\left|M_{\sigma}(q)\right|_{L^{\infty}(\partial \mathcal{O})}+\mathscr{Z}(v, h, \mathcal{O}, \mu, q), \\
& \mathscr{Z}=3^{(8 / 5)} \\
& \times\left(\frac{3}{2}\right)^{(28 / 5)} \frac{(2 \pi)^{(1 / 4)}}{h_{0}^{3}} \operatorname{diam} \mathcal{O}\left(|v| h_{1}|\mathcal{O}|^{1 / 5}\right. \\
& \left.+h_{1}^{3}\left|\nabla M_{\sigma}(q)\right|_{L^{5}(G)}\right) \\
& \times|\mathcal{O}|^{(7 / 5)} .
\end{aligned}
$$

Proof. The function $z=w-G$ satisfies

$$
\begin{gathered}
\operatorname{div}\left(h^{3} \nabla z\right)=v \frac{\partial h}{\partial x}+\operatorname{div}\left(h^{3} \nabla M_{\sigma}(q)\right) \quad \text { in } \mathcal{O} \subset \mathbf{R}^{2}, \\
z=0 \quad \text { on } \partial \mathcal{O} .
\end{gathered}
$$

Next we introduce the embedding constant for $W_{0}^{1,2}(\mathcal{O}) \subset$ $L^{r}(\mathcal{O})$ denoted $M_{r}$, such that

$$
|v|_{L^{r}(\mathcal{O})} \leq M_{r}|\nabla v|_{L^{2}(\mathcal{O})}, \quad \forall v \in H_{0}^{1}(\mathcal{O}) .
$$

That constant can be estimated as

$$
M_{r} \leq \frac{1}{2}(\operatorname{diam} \mathcal{O})^{2}|\mathcal{O}|^{1 / r-1 / 2}\left(\frac{r+2}{2}\right)^{((r+2) / 2 r)} \sqrt{2 \pi} .
$$

See, for example, [20, Lemma 1]. Next we define the sequence

$$
\lambda_{k+1}=3\left(\frac{\lambda_{k}}{2}+1\right), \quad \lambda_{1}=2 .
$$

Easy computation yields

$$
\lambda_{k}=8\left(\frac{3}{2}\right)^{k-1}-6
$$

Let

$$
z^{+}(x, y)=\max \{z(x, y), 0\} .
$$

We test (A.5) with $\left(z^{+}\right)^{1+\lambda_{k+1}}$ and get

$$
\begin{aligned}
& \int_{\mathscr{O}} h^{3} \nabla z^{+} \nabla\left(z^{+}\right)^{1+\lambda_{k+1}} \\
& =\frac{1+\lambda_{k+1}}{\left(1+\lambda_{k+1} / 2\right)^{2}} \int_{\mathcal{O}} h^{3}\left|\nabla\left(z^{+}\right)^{1+\left(\lambda_{k+1} / 2\right)}\right|^{2} \\
& \quad=\int_{\mathcal{O}}\left[v h \frac{\partial}{\partial x}\left(z^{+}\right)^{1+\lambda_{k+1}}+h^{3} \nabla M_{\sigma}(q) \nabla\left(z^{+}\right)^{1+\lambda_{k+1}}\right] .
\end{aligned}
$$

For the left-hand side, we get the lower bound

$$
h_{0}^{3} \frac{1+\lambda_{k+1}}{\left(1+\lambda_{k+1} / 2\right)^{2}}\left|\nabla\left(z^{+}\right)^{1+\left(\lambda_{k+1} / 2\right)}\right|_{L^{2}(\mathcal{O})}^{2} .
$$

We estimate the terms on the right-hand side using the same idea

$$
\begin{aligned}
\int_{\mathscr{O}} h^{3} \nabla & M_{\sigma}(q) \nabla\left(z^{+}\right)^{1+\lambda_{k+1}} \\
& \leq h_{1}^{3}\left|\nabla M_{\sigma}(q)\right|_{L^{5}(\mathcal{O})}\left|\nabla\left(z^{+}\right)^{1+\lambda_{k+1}}\right|_{L^{5 / 4}(\mathcal{O})^{\prime}} \\
\int_{\mathscr{O}} h v & \frac{\partial}{\partial x}\left(z^{+}\right)^{1+\lambda_{k+1}} \\
\leq & h_{1}|v||\mathcal{O}|^{1 / 5}\left|\nabla\left(z^{+}\right)^{1+\lambda_{k+1}}\right|_{L^{5 / 4}(\mathcal{O})^{\circ}}
\end{aligned}
$$

Thus, it remains to estimate $\left|\nabla\left(z^{+}\right)^{1+\lambda_{k+1}}\right|_{L^{5 / 4}(\mathcal{O})}$. We have

$$
\begin{aligned}
\mid \nabla & \left.\left(z^{+}\right)^{1+\lambda_{k+1}}\right|_{L^{5 / 4}(\mathcal{O})} ^{5 / 4} \\
= & \left(1+\lambda_{k+1}\right)^{5 / 4} \int_{\mathcal{O}}\left(z^{+}\right)^{\left(5 \lambda_{k+1} / 4\right)}\left|\nabla z^{+}\right|^{5 / 4} \\
= & \left(\frac{1+\lambda_{k+1}}{1+\left(\lambda_{k+1} / 2\right)}\right)^{5 / 4} \int_{\mathcal{O}}\left|\nabla\left(z^{+}\right)^{1+\left(\lambda_{k+1} / 2\right)}\right|^{5 / 4}\left|z^{+}\right|^{\left(5 \lambda_{k+1} / 8\right)} \\
\leq & {\left[\text { Hölders inequality with } p=\frac{8}{5}, p^{\prime}=\frac{8}{3}\right] } \\
\leq & \left(\frac{1+\lambda_{k+1}}{1+\left(\lambda_{k+1} / 2\right)}\right)^{5 / 4}\left(\int_{\mathcal{O}}\left|\nabla\left(z^{+}\right)^{1+\left(\lambda_{k+1} / 2\right)}\right|^{2}\right)^{5 / 8} \\
& \times\left(\int_{\mathcal{O}}\left(z^{+}\right)^{(5 / 3) \lambda_{k+1}}\right)^{3 / 8} \\
= & {\left[\text { due to (A.9) } \frac{5}{3} \lambda_{k+1}=5\left(1+\frac{\lambda_{k}}{2}\right)\right.} \\
& \left.\frac{3}{8}=\frac{1}{4} \frac{\lambda_{k+1}}{2+\lambda_{k}}\right] \\
= & \left(\frac{1+\lambda_{k+1}}{1+\left(\lambda_{k+1} / 2\right)}\right)^{5 / 4}\left|\nabla\left(z^{+}\right)^{1+\left(\lambda_{k+1} / 2\right)}\right|_{L^{2}(\mathcal{O})}^{5 / 4} \\
& \times\left|\left(z^{+}\right)^{1+\left(\lambda_{k} / 2\right)}\right|_{L^{5}(\mathcal{O})}^{\left(\lambda_{k+1} /\left(2+\lambda_{k}\right)\right)(5 / 4)} .
\end{aligned}
$$

Combining with (A.14) and (A.13), we get

$$
\begin{aligned}
\left|\nabla\left(z^{+}\right)^{1+\left(\lambda_{k+1} / 2\right)}\right|_{L^{2}(\mathcal{O})} & \\
\leq & h_{0}^{-3}\left(1+\frac{\lambda_{k+1}}{2}\right)\left|\left(z^{+}\right)^{1+\left(\lambda_{k} / 2\right)}\right|_{L^{5}(\mathcal{O})}^{\left(\left(\lambda_{k+1}\right) /\left(2+\lambda_{k}\right)\right)} \\
\quad & \times\left(h_{1}^{3}\left|\nabla M_{\sigma}(q)\right|_{L^{5}(\mathcal{O})}+h_{1}|v||\mathcal{O}|^{1 / 5}\right)
\end{aligned}
$$




$$
\begin{aligned}
\leq & M_{5}^{\left(\left(\lambda_{k+1}\right) /\left(2+\lambda_{k}\right)\right)} h_{0}^{-3}\left(1+\frac{\lambda_{k+1}}{2}\right) \\
& \times\left|\nabla\left(z^{+}\right)^{1+\left(\lambda_{k} / 2\right)}\right|_{L^{2}(\mathcal{O})}^{\left(\left(\lambda_{k+1}\right) /\left(2+\lambda_{k}\right)\right)} \\
& \times\left(h_{1}^{3}\left|\nabla M_{\sigma}(q)\right|_{L^{5}(\mathcal{O})}+h_{1}|v||\mathcal{O}|^{1 / 5}\right) .
\end{aligned}
$$

We recall that

$$
\frac{\lambda_{k+1}}{2+\lambda_{k}}=\frac{3}{2}
$$

and define

$$
\alpha=M_{5}^{(3 / 2)} h_{0}^{-3}\left(h_{1}^{3}\left|\nabla M_{\sigma}(q)\right|_{L^{5}(\mathcal{O})}+h_{1}|v||\mathcal{O}|^{1 / 5}\right)
$$

as well as

$$
\sigma_{k}=\frac{1}{\alpha}\left|\nabla\left(z^{+}\right)^{1+\left(\lambda_{k} / 2\right)}\right|_{L^{2}(\mathcal{O})}^{\left(2 /\left(2+\lambda_{k}\right)\right)} .
$$

Then (A.16) implies

$$
\sigma_{k+1} \leq\left(1+\frac{\lambda_{k+1}}{2}\right)^{\left(2 /\left(2+\lambda_{k}\right)\right)} \sigma_{k}^{\left(\left(\lambda_{k+1}\right) /\left(2+\lambda_{k+1}\right)\right)} .
$$

Taking the logarithm, we arrive at

$$
\log \sigma_{k+1} \leq \frac{\log \left(1+\left(\lambda_{k+1} / 2\right)\right)}{1+\left(\lambda_{k+1} / 2\right)}+\frac{\lambda_{k+1}}{2+\lambda_{k+1}} \log \sigma_{k} .
$$

We first notice that

$$
\frac{\lambda_{k+1}}{2+\lambda_{k+1}}<1
$$

and then

$$
1+\frac{\lambda_{k}}{2}=4\left(\frac{3}{2}\right)^{k-1}-2>\left(\frac{3}{2}\right)^{k}
$$

Since the function $x \mapsto(\log x / x)$ is decreasing for $x>e$, we have

$$
\begin{aligned}
\frac{\log \left(1+\left(\lambda_{k} / 2\right)\right)}{1+\left(\lambda_{k} / 2\right)} & \leq \frac{\log \left[(3 / 2)^{k}\right]}{(3 / 2)^{k}} \\
& =k \log \frac{3}{2}\left(\frac{2}{3}\right)^{k}, \quad \forall k \geq 3 .
\end{aligned}
$$

Then,

$$
\begin{aligned}
\log \sigma_{k+1} & \leq(k+1) \log \frac{3}{2}\left(\frac{2}{3}\right)^{k}+\log \sigma_{k} \\
& \leq \log \frac{3}{2} \sum_{j=2}^{k+1} j\left(\frac{2}{3}\right)^{j}+\log \sigma_{1} \leq 8 \log \frac{3}{2}+\log \sigma_{1} .
\end{aligned}
$$

Finally

$$
\sigma_{k+1} \leq\left(\frac{3}{2}\right)^{8} \sigma_{1}
$$

Now it remains to estimate $\sigma_{1}$. From the definition we see that

$$
\sigma_{1}=\frac{1}{\alpha}\left|\nabla\left(z^{+}\right)^{2}\right|_{L^{2}(\mathcal{O})}^{1 / 2}
$$

To estimate $\sigma_{1}$, we proceed as before and test (A.5) with $\left(z^{+}\right)^{3}$. We get

$$
\begin{aligned}
& \frac{3}{4} \int_{\mathscr{O}} h^{3}\left|\nabla\left(z^{+}\right)^{2}\right|^{2} \\
& =\int_{\mathcal{O}}\left(v h \frac{\partial\left(z^{+}\right)^{3}}{\partial x}+h^{3} \nabla M_{\sigma}(q) \nabla\left(z^{+}\right)^{3}\right) \\
& \leq\left(|v| h_{1}|\mathcal{O}|^{1 / 5}+h_{1}^{3}\left|\nabla M_{\sigma}(q)\right|_{L^{5}(\mathcal{O})}\right)\left|\nabla\left(z^{+}\right)^{3}\right|_{L^{5 / 4}(\mathcal{O})} \\
& \leq\left(|v| h_{1}|\mathcal{O}|^{1 / 5}+h_{1}^{3}\left|\nabla M_{\sigma}(q)\right|_{L^{5}(\mathcal{O})}\right) \\
& \quad \times \frac{3}{2}\left|\nabla\left(z^{+}\right)^{2}\right|_{L^{2}(\mathcal{O})}\left|z^{+}\right|_{L^{10 / 3}(\mathcal{O})^{\circ}}
\end{aligned}
$$

Thus

$$
\begin{aligned}
& \left|\nabla\left(z^{+}\right)^{2}\right|_{L^{2}(\mathcal{O})} \leq \frac{2}{h_{0}^{3}}\left(|v| h_{1}|\mathcal{O}|^{1 / 5}\right. \\
& \left.+h_{1}^{3}\left|\nabla M_{\sigma}(q)\right|_{L^{5}(\mathcal{G})}\right) M_{(10 / 3)}\left|\nabla z^{+}\right|_{L^{2}(\mathcal{O})}
\end{aligned}
$$

Finally, testing (A.5) with $z^{+}$, we get

$$
\int_{\mathscr{O}} h^{3}\left|\nabla z^{+}\right|^{2}=\int_{\mathscr{O}}\left(v h \frac{\partial z^{+}}{\partial x}+h^{3} \nabla M_{\sigma}(q) \nabla z^{+}\right)
$$

so that

$$
\begin{aligned}
\left|\nabla z^{+}\right|_{L^{2}(\mathcal{O})} & \leq \frac{1}{h_{0}^{3}}\left(|v| h_{1}|\mathcal{O}|^{1 / 2}+h_{1}^{3}\left|\nabla M_{\sigma}(q)\right|_{L^{2}(\mathcal{O})}\right) \\
& \leq|\mathcal{O}|^{3 / 10}\left(|v| h_{1}|\mathcal{O}|^{1 / 5}+h_{1}^{3}\left|\nabla M_{\sigma}(q)\right|_{L^{5}(\mathcal{O})}\right) .
\end{aligned}
$$

Combining (A.29) with (A.31) and (A.26) gives

$$
\begin{aligned}
\sigma_{k+1} \leq & \left(\frac{3}{2}\right)^{8} \frac{1}{\alpha} \frac{\sqrt{2}}{h_{0}^{3}} \\
& \times\left(|v| h_{1}|\mathcal{O}|^{1 / 5}\right. \\
& \left.+h_{1}^{3}\left|\nabla M_{\sigma}(q)\right|_{L^{5}(\mathcal{O})}\right) M_{(10 / 3)}^{1 / 2}|\mathcal{O}|^{3 / 2} .
\end{aligned}
$$

Since

$$
\sigma_{k+1} \geq \frac{1}{\alpha M_{2}^{\left(2 /\left(2+\lambda_{\mathrm{k}}\right)\right)}}\left|z^{+}\right|_{L^{2+\lambda_{k}(\mathcal{O})}},
$$


we have arrived to

$$
\begin{aligned}
\left|z^{+}\right|_{L^{2+\lambda_{k}(\mathcal{O})}} \leq & \left(\frac{3}{2}\right)^{8} M_{2}^{\left(2 /\left(2+\lambda_{k}\right)\right)} \frac{\sqrt{2}}{h_{0}^{3}} \\
& \times\left(|v| h_{1}|\mathcal{O}|^{1 / 5}\right. \\
& \left.\quad+h_{1}^{3}\left|\nabla M_{\sigma}(q)\right|_{L^{5}(\mathcal{O})}\right) M_{(10 / 3)}^{1 / 2}|\mathcal{O}|^{3 / 2} .
\end{aligned}
$$

Since $\lim _{k \rightarrow \infty} \lambda_{k}=\infty$, we get

$$
\begin{aligned}
\left|z^{+}\right|_{L^{\infty}(\mathcal{O})} \leq & \left(\frac{3}{2}\right)^{8} \frac{\sqrt{2}}{h_{0}^{3}} \\
& \times\left(|v| h_{1}|\mathcal{O}|^{1 / 5}+h_{1}^{3}\left|\nabla M_{\sigma}(q)\right|_{L^{5}(\mathcal{O})}\right) M_{(10 / 3)}^{1 / 2}|\mathcal{O}|^{3 / 2}
\end{aligned}
$$

Finally (A.8) implies (A.3).

\section{Acknowledgment}

This work was supported by MZOS grant 037-0372787-2797.

\section{References}

[1] O. Reynolds, "On the theory and application and its application to Mr. Beauchamp tower's experiments, Including and experimental determination of the viscosity of olive oil," Philosophical Transactions of the Royal Society, vol. 117, pp. 157-234, 1886.

[2] G. G. Stokes, "Notes on hydrodynamics. On the dynamical equations," Cambridge and Dublin Mathematical Journal III, pp. 121-127, 1848.

[3] P. W. Brifgman, "The viscosity of liquids under pressure," Proceedings of the National Academy of Sciences of the United States of America, vol. 11, no. 10, pp. 603-606, 1925.

[4] A. Z. Szeri, Fluid Film Lubrication, Cambridge University Press, New York, NY, USA, 1998.

[5] E. K. Gatcombe, "Lubrication characteristics of involute spurgears-a theoretical investigation," Transactions of the American Society of Mechanical Engineers, vol. 67, pp. 177-181, 1945.

[6] C. Barus, "Isotherms, isopiestics and isometrics relative to viscosity," American Journal of Science, vol. 45, pp. 87-96, 1893.

[7] W. R. Jones, "Pressure viscosity measurement for several lubricants," ASLE Transactions, vol. 18, pp. 249-262, 1975.

[8] J. Hron, J. Málek, and K. R. Rajagopal, "Simple flows of fluids with pressure-dependent viscosities," Proceedings of the Royal Society A, vol. 457, no. 2011, pp. 1603-1622, 2001.

[9] C. J. A. Roelands, Correlation aspects of the viscosity-pressure relationship of lubricating oils [Ph.D. thesis], Delft University of Technology, Delft, The Netherlands, 1966.

[10] K. R. Rajagopal and A. Z. Szeri, "On an inconsistency in the derivation of the equations of elastohydrodynamic lubrication," Proceedings of the Royal Society A, vol. 459, no. 2039, pp. 27712786, 2003.

[11] M. Renardy, "Some remarks on the Navier-Stokes equations with a pressure-dependent viscosity," Communications in Partial Differential Equations, vol. 11, no. 7, pp. 779-793, 1986.

[12] F. Gazzola and P. Secchi, "Some results about stationary NavierStokes equations with a pressure-dependent viscosity," in Proceedings of the International Conference on Navier-Stokes Equations, vol. 388 of Pitman Research Notes in Mathematics Series, pp. 174-183, Varenna, Italy, 1998.
[13] E. Marusic-Paloka, "An analysis of the Stokes system with pressure dependent viscosity," In press.

[14] S. Marušić and E. Marušić-Paloka, "Two-scale convergence for thin domains and its applications to some lower-dimensional models in fluid mechanics," Asymptotic Analysis, vol. 23, no. 1, pp. 23-57, 2000.

[15] D. Gilbarg and N. S. Trudinger, Elliptic Partial Differential Equations of Second Order, Springer, Berlin, Germany, 2001.

[16] M. Kane and B. Bou-Said, "Comparison of homogenization and direct techniques for the treatment of roughness in incompressible lubrication," Journal of Tribology, vol. 126, no. 4, pp. 733-737, 2004.

[17] M. Jai, "Homogenization and two-scale convergence of the compressible Reynolds lubrication equation modelling the flying characteristics of a rough magnetic head over a rough rigiddisk surface," RAIRO Modélisation Mathématique et Analyse Numérique, vol. 29, no. 2, pp. 199-233, 1995.

[18] P. Wall, "Homogenization of Reynolds equation by two-scale convergence," Chinese Annals of Mathematics B, vol. 28, no. 3 , pp. 363-374, 2007.

[19] A. Bensoussan, J.-L. Lions, and G. Papanicolaou, Asymptotic Analysis for Periodic Structures, North-Holland, Amsterdam, The Netherlands, 1978.

[20] E. Marušić-Paloka and A. Mikelić, "The derivation of a nonlinear filtration law including the inertia effects via homogenization," Nonlinear Analysis: Theory, Methods \& Applications, vol. 42, no. 1, pp. 97-137, 2000. 


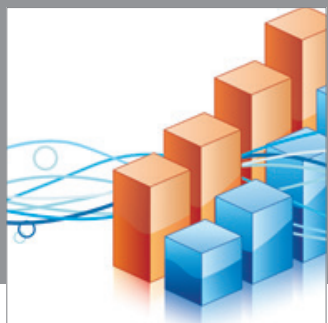

Advances in

Operations Research

mansans

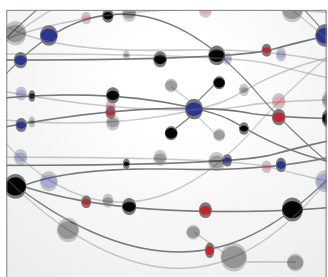

The Scientific World Journal
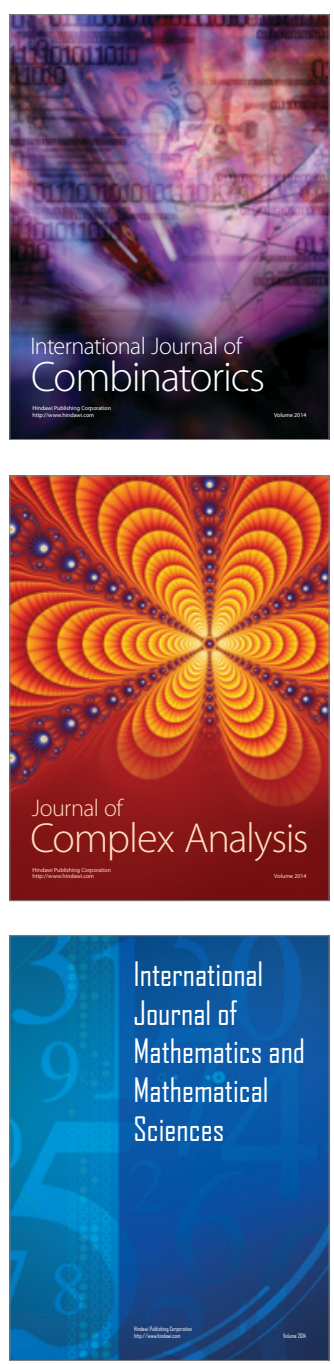
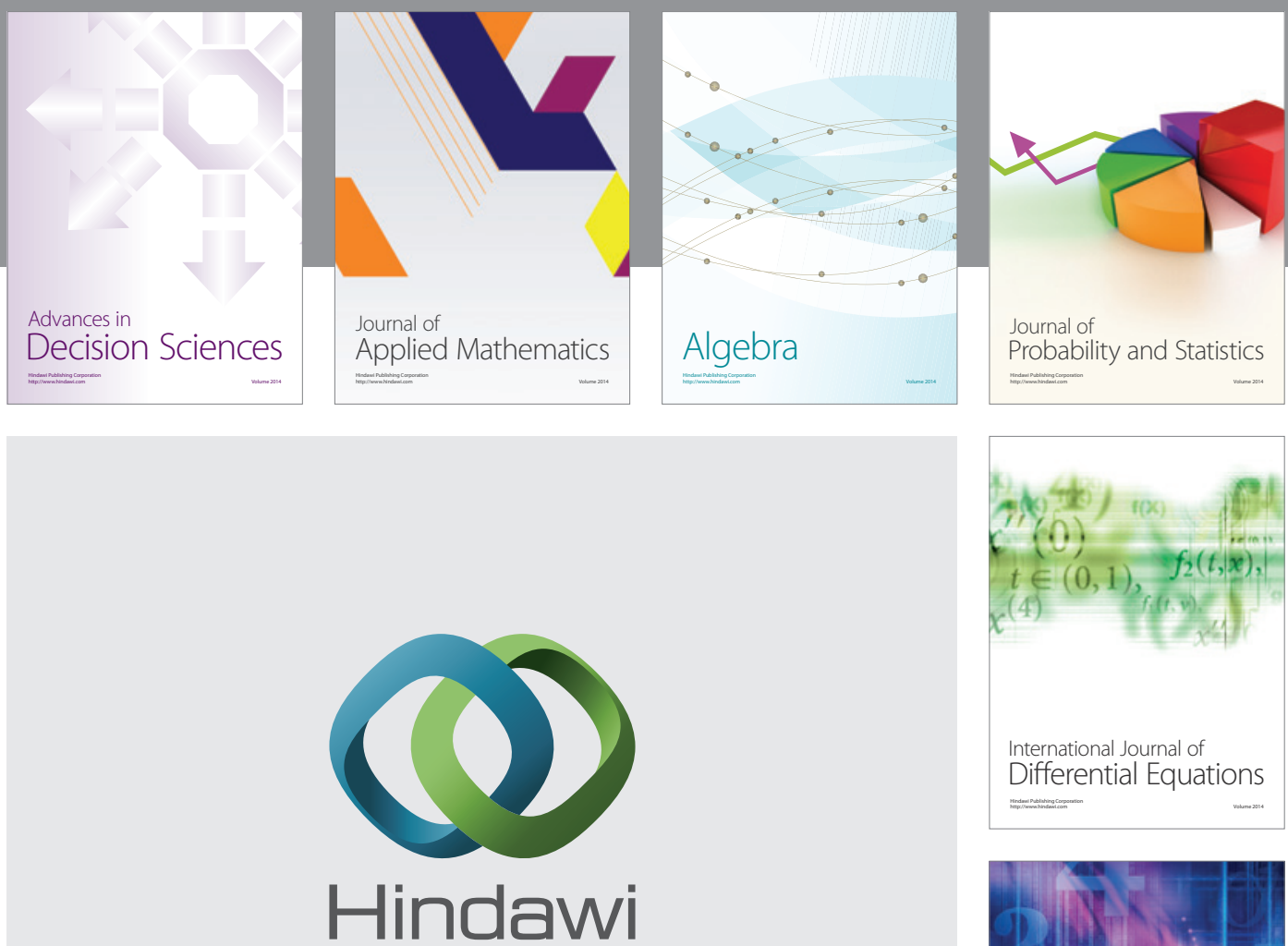

Submit your manuscripts at http://www.hindawi.com
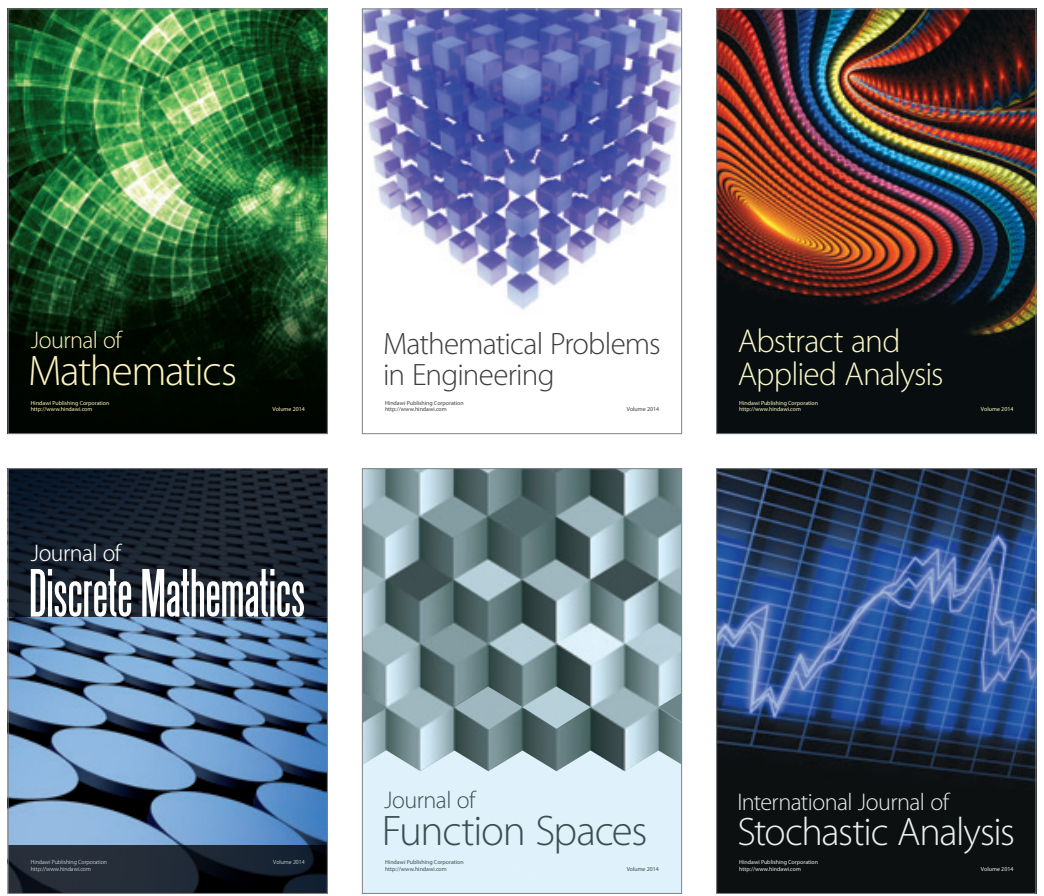

Journal of

Function Spaces

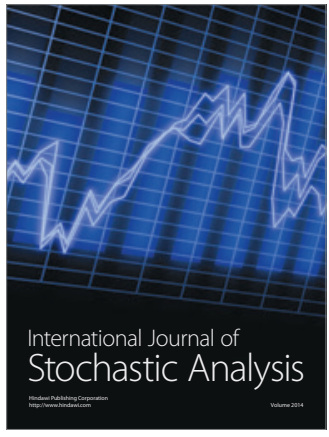

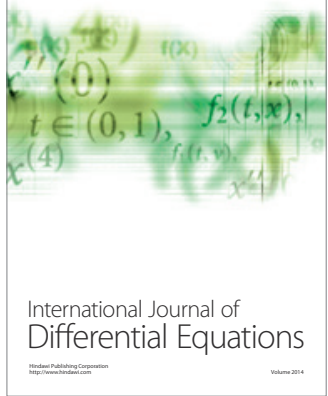
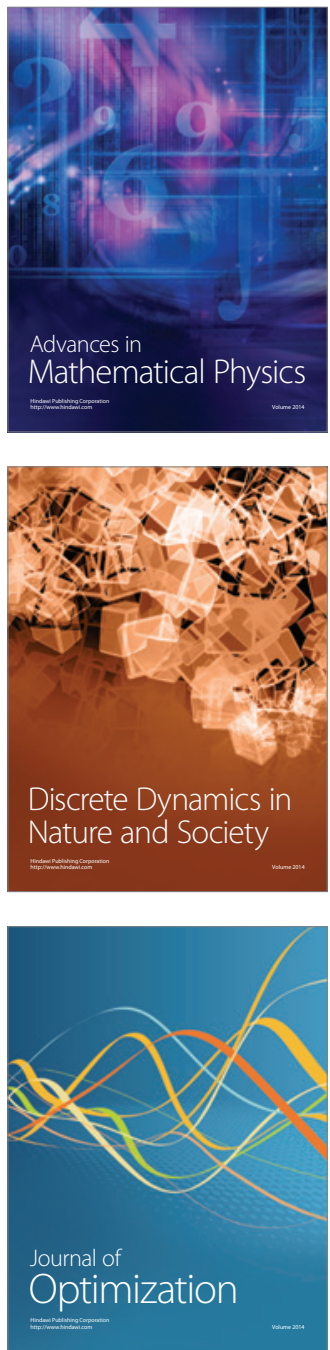